This item was submitted to Loughborough's Research Repository by the author.

Items in Figshare are protected by copyright, with all rights reserved, unless otherwise indicated.

\title{
Unmanned aerial vehicles: control methods and future challenges
}

PLEASE CITE THE PUBLISHED VERSION

https://doi.org/10.1109/jas.2022.105410

\section{PUBLISHER}

Institute of Electrical and Electronics Engineers (IEEE)

VERSION

AM (Accepted Manuscript)

\section{PUBLISHER STATEMENT}

(c) 2022 IEEE. Personal use of this material is permitted. Permission from IEEE must be obtained for all other uses, in any current or future media, including reprinting/republishing this material for advertising or promotional purposes, creating new collective works, for resale or redistribution to servers or lists, or reuse of any copyrighted component of this work in other works.

\section{LICENCE}

All Rights Reserved

\section{REPOSITORY RECORD}

Zuo, Zongyu, Cunjia Liu, Qing-Long Han, and Jiawei Song. 2022. "Unmanned Aerial Vehicles: Control Methods and Future Challenges". Loughborough University. https://hdl.handle.net/2134/19102517.v1. 


\title{
Unmanned Aerial Vehicles: Control Methods and Future Challenges
}

\author{
Zongyu Zuo, Senior Member, IEEE, Cunjia Liu, Member, IEEE, Qing-Long Han, Fellow, IEEE, and Jiawei Song
}

\begin{abstract}
With the rapid development of computer technology, automatic control technology and communication technology, research on unmanned aerial vehicles (UAVs) has attracted extensive attention from all over the world during the last decades. Particularly due to the demand of various civil applications, the conceptual design of $\mathrm{UAV}$ and autonomous flight control technology have been promoted and developed mutually. This paper is devoted to providing a brief review of the UAV control issues, including motion equations, various classical and advanced control approaches. The basic ideas, applicable conditions, advantages and disadvantages of these control approaches are illustrated and discussed. Some challenging topics and future research directions are raised.
\end{abstract}

Index Terms-Aircraft control, disturbance rejection, pathfollowing, trajectory tracking, unmanned aerial vehicle.

\section{NOMENCLATURE}

$\mathcal{E} \quad$ Earth-surface inertial frame

$O_{E} \quad$ Origin of Frame $\mathcal{E}$

$x_{E}, y_{E}, z_{E} \quad$ Axis of Frame $\mathcal{E}$

$\mathcal{B} \quad$ Aircraft-body frame

$O \quad$ Origin of Frame $\mathcal{B}$

$x, y, z \quad$ Axis of Frame $\mathcal{B}$

$W \quad$ Wind frame

$x_{W}, y_{W}, z_{W} \quad$ Axis of Frame $\mathcal{W}$

$\mathcal{P} \quad$ Flight-path coordinate frame

$x_{P}, y_{P}, z_{P} \quad$ Axis of Frame $\mathcal{P}$

$u, v, w \quad$ Inertial velocities

$a_{x}, a_{y}, a_{z} \quad$ Body axis accelerations

$\phi, \theta, \psi \quad$ Euler angles

This article has been accepted for publication in a future issue of this journal, but has not been fully edited. Content may change prior to final publication.

This work was supported by the National Natural Science Foundation of China (62073019). Recommended by Associate Editor Xiaoxiang Na. (Corresponding author: Qing-Long Han.)

Citation: Z. Zuo, C. J. Liu, H.-L. Han, and J. Song, "Unmanned aerial vehicles: Control methods and future challenges," IEEE/CAA J. Autom. Sinica, 2022, DOI: 10.1109/JAS.2022.105410

Z. Zuo and J. Song are with the Seventh Research Division, Beihang University (BUAA), Beijing 100191, China (e-mail: zzybobby@buaa.edu.cn, songjw@buaa.edu.cn).

C. Liu is with Department of Aeronautical and Automotive Engineering, Loughborough University, Leicestershire, LE11 3TU, United Kingdom (email: c.liu5@lboro.ac.uk).

Q.-L. Han is with School of Science, Computing and Engineering Technologies, Swinburne University of Technology, Melbourne, VIC 3122, Australia (e-mail: qhan@swin.edu.au).

Color versions of one or more of the figures in this paper are available online at http://ieeexplore.iee.org.

Digital Object Identifier 10.1109/JAS.2022.105410

\author{
$p, q, r \quad$ Angular velocities measured in the frame $\mathcal{B}$ \\ $\alpha \quad$ Attack angle \\ $\beta \quad$ Sideslip angle \\ $\chi \quad$ Heading angle \\ $\gamma \quad$ Flight-path angle \\ $\mu \quad$ Bank angle \\ $m \quad$ Mass of aircraft \\ $I_{x}, I_{y}, I_{z} \quad$ Moments of inertia \\ $I_{x y}, I_{x z}, I_{y z} \quad$ Product of inertia \\ $F_{i}^{j} \quad$ The $i$-axis components of principle vector $F$ \\ acting on the aircraft projected in the frame $j$, \\ $i=\{x, y, z\}, j=\{\mathcal{B}, \mathcal{W}, \mathcal{P}\}$ \\ $\begin{array}{ll}M_{i}^{B} & \text { The } i \text {-axis components of principal moment } M \\ \text { acting on the aircraft projected in the frame } \mathcal{B}\end{array}$
}

\section{INTRODUCTION}

A unmanned aerial vehicle (UAV) (or unpiloted aerial vehicle) is commonly known as an aircraft without a human pilot onboard that can fly autonomously. It is the powered aerial vehicle that uses aerodynamics and propulsion to provide vehicle lift and control forces. Compared with crewed aircraft, UAVs were originally used for missions usually dull, dirty or dangerous for humans or cases where the small size of the aircraft restrict the presence of a pilot. Although initially reviewed as military devices, UAVs rapidly find more civil applications including aerial photography, payload deliveries, smart agriculture, surveillance, infrastructure inspections, and drone racing, to name a few.

UAVs can be usually categorized into two main classes: fixed-wing UAVs and rotorcraft UAVs. The fixed-wing UAV requires relative velocity for the production of aerodynamic forces, thus is more aerodynamically efficient, but may need a runway for take-off and landing. The rotorcraft UAV uses rotary wings to produce the thrust force for motion [1], which leads to the vertical take-off and landing (VTOL) capability. The combination of fixed-wing and VTOL features motivates to many hybrid UAV configurations, which become prevalent in recent years [2]. Correspondingly, the control design for different kinds of UAVs also varies.

This survey is written in a control-oriented fashion. On the one hand, the survey introduces basic elements in flight control design. On the other hand, the survey may serve as a catalogue of control design methods for researchers and practitioners in the community.

\section{MAIN ISSUES IN CONTROL}

The development of various new types of UAVs created a 
great need for autopilot systems. However, to achieve multimission and high flight quality of aircraft, it is very challenging for flight control system design. These issues include, but are not limited to:

- In essence, classical control design relies heavily on the understanding of the physics of flight and provides a designer with clues to see how the dynamic performance is modified. Although classical control theory relates very closely to the physics of the control problem, it is difficult to handle the coupling multiple loops design task [3]. In other words, the classical one-loop-at-a-time design does not guarantee success when more loops are added and coupled.

- In contrast to classical techniques, modern control techniques (e.g., eigenstructure assignment techniques [4], dynamic inversion [5]) provide a systematic way to design multi-loop controllers by closing all the feedback loops simultaneously and yield the desired performance. However, these model control techniques usually require the exact knowledge of aircraft models and are sensitive to modeling errors and external disturbances. Unfortunately, the exact model of aircraft like helicopters is difficult to obtain, especially aerodynamic parameters, which prohibits the practicality of modern control techniques.

- There exists an increasing interest in adaptive control of aircraft. The progress in systems theory leads to fundamental theory for the development of stable adaptive control architectures [6]. The available adaptive flight control algorithms, however, are unable to guarantee the closed-loop robustness because the bandwidth of the closed-loop system cannot be prescribed [7]. In addition, the system output has overly poor transient tracking behaviour before the ideal asymptotic convergence is achieved [8]. Thus, robustness and stability issues of adaptive flight controllers need to be considered simultaneously in design [9].

- Disturbances and uncertainties are the factors which may cause the unsatisfactory performance. It is very important to design flight controllers accounting for disturbances, such as wind gusts, sensor measurement noise, and modeling errors. The modern robust design techniques (e.g., $H_{\infty}$ design [10]) provide performance robustness which is the ability to ensure satisfactory performance even if the aircraft may be subject to disturbances. However, it is usually difficult to obtain the upper-bounds of various disturbances and a controller with the desired structure.

\section{KINEMATICS AND DYNAMICS OF AIRCRAFT MOTION}

\section{A. Coordinate Systems}

In studying unmanned aircraft systems, it is important to understand how an aircraft is oriented with respect to the earth. It is necessary to use several different coordinate systems to describe the position and the orientation of the aircraft in an easy manner. The coordinate systems of interest include an earth-fixed frame, a body-fixed frame, a wind frame, and a flight-path coordinate frame, as shown in Fig. 1.

Definition 1: Frame $\mathcal{E}=\left\{O_{E} x_{E} y_{E} z_{E}\right\}$ denotes an earthsurface inertial frame with its origin $O_{E}$ at the defined home location, $x_{E}$ axis positive in the direction of north, $y_{E}$ axis

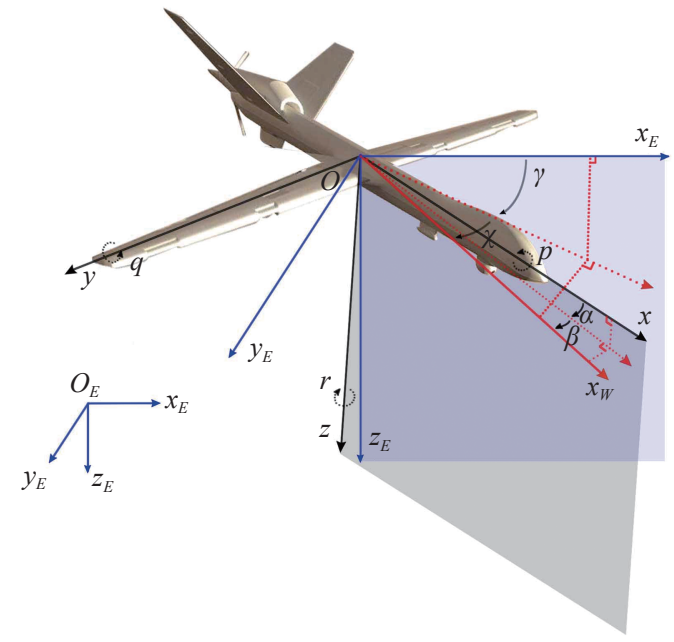

Fig. 1. Coordinate systems.

positive in the direction of east and $z_{E}$ axis down. This coordination system is also known as a north-east-down (NED) reference frame.

Definition 2: Frame $\mathcal{B}=\{O x y z\}$ denotes an aircraft-body frame with its origin $O$ at the center of mass of an aircraft, $x$ axis positive out the nose of the airframe, $y$ axis positive out the right wing and $z$ axis positive below the aircraft.

Definition 3: Frame $\mathcal{W}=\left\{O x_{W} y_{W} z_{W}\right\}$ denotes a wind frame with its origin $O$ at the center of mass of an aircraft, $x_{W}$ axis positive in the direction of the velocity vector of the aircraft relative to the air, i.e. the airspeed vector, $z_{W}$ axis positive below the aircraft in the plane of symmetry of the aircraft, $y_{W}$ positive determined by the right hand rule, i.e. to the right.

Definition 4: Frame $\mathcal{P}=\left\{O x_{P} y_{P} z_{P}\right\}$ denotes a flight-path coordinate frame with its origin $O$ at the center of mass of the aircraft, $x_{P}$ axis positive in the direction of the velocity vector of the aircraft relative to the ground, i.e. the ground vector, $z_{P}$ axis positive below the aircraft in the plane of symmetry of the aircraft, $y_{P}$ positive determined by the right hand rule.

Remark 1: Note that if wind is not taken into account, the frame $\mathcal{P}$ coincides with the frame $\mathcal{W}$. In flight control system design, wind is regarded as an external disturbance rather than a system state. In what follows, the kinematic and dynamic equations are established in the absence of wind.

Remark 2: The earth-surface inertial frame is convenient to express translational kinematics. The aircraft-body frame is especially convenient in the sense that the inertial parameters and the force of thrust remain fixed. The wind frame facilitates the expressions of the aerodynamic forces and moments acting on an aircraft.

In addition to defining the coordinate systems, the relative orientation of these frames can be determined and expressed in various forms, such as rotation matrices, Euler angles, unit quaternions. In particular, various Euler angles relating to these coordinate systems are important to the flight dynamics of UAVs. In flight dynamics, roll, pitch, and yaw Euler angles (i.e., $\phi, \theta$, and $\psi$, respectively) are commonly used to parameterize the rotation from the frame $\mathcal{E}$ to the frame $\mathcal{B}$, while the sideslip angle $\beta$ and the angle of attack $\alpha$ are commonly used to perform the transformation from the frame 
$\mathcal{W}$ to the frame $\mathcal{B}$. Analogously, to obtain the frame $\mathcal{W}$ from the frame $\mathcal{E}$, a set of Euler angles, namely, the heading angle $\chi$, the flight-path angle $\gamma$, and the bank angle $\mu$ are frequently utilized.

\section{B. Kinematic Equations}

The kinematics of a UAV includes the translational and the rotational equation of motion. The following assumption is usually made in the existing literature in developing navigation, guidance, and control strategies for UAVs [11], [12].

Assumption 1: The Earth's rotation and revolution movement can be ignored so that the frame $\mathcal{E}$ can be regarded as an inertial reference frame. In addition, a flat earth model is assumed, which is appropriate for small UAVs.

1) Translational kinematics: The translational velocity of a UAV is commonly expressed in terms of velocity components along each of axes in an aircraft-body coordinate frame $\mathcal{B}$. Let $V^{B}=[u, v, w]^{T}$ be the inertial velocity of a UAV measured along the axes of the frame $\mathcal{B}$, and $\boldsymbol{p}^{E}=\left[x_{E}, y_{E}, z_{E}\right]^{T}$ be the inertial position projected onto the axes of the frame $\mathcal{E}$. The translational equations of an aircraft (also known as navigation equations) are described by

$$
\left\{\begin{aligned}
\dot{x}_{E}= & u \cos \theta \cos \psi+v(\sin \phi \sin \theta \cos \psi-\cos \phi \sin \psi) \\
& +w(\sin \phi \sin \psi+\cos \phi \sin \theta \cos \psi) \\
\dot{y}_{E}= & u \cos \theta \sin \psi+v(\sin \phi \sin \theta \sin \psi+\cos \phi \cos \psi) \\
& -w(\sin \phi \cos \psi-\cos \phi \sin \theta \sin \psi) \\
\dot{z}_{E}= & -u \sin \theta+v \sin \phi \cos \theta+w \cos \phi \cos \theta
\end{aligned}\right.
$$

2) Rotational kinematics: In many flight dynamics applications, roll, pitch, and yaw Euler angles, as one of conventions, are usually used to describe the aircraft orientation relative to the frame $\mathcal{E}$. Let $\boldsymbol{\Omega}=[p, q, r]^{T}$ be the angular velocity vector of a UAV measured in the frame $\mathcal{B}$. The rotational equations of an aircraft in terms of the three Euler angles are given as

$$
\left\{\begin{array}{l}
\dot{\phi}=p+(r \cos \phi+q \sin \phi) \tan \theta \\
\dot{\theta}=q \cos \phi-r \sin \phi \\
\dot{\psi}=\frac{1}{\cos \theta}(r \cos \phi+q \sin \phi)
\end{array}\right.
$$

Remark 3: It should be noted that the rotational kinematic description (2) has a singularity point at $\theta=\pi / 2$. This imposes certain limitations on the application of the Euler angle for vehicles operating in a large range. Fortunately, many UAVs, such as fixed-wing aircraft and helicopters, usually operate within a small variation of attitude. To enlarge the operation profile, one can resort to unit quaternion [13] or modified Rodrigues parameters (MRPs) [14]. However, such singularities occur in any 3-D representation of $\mathrm{SO}(3)$ [15]. More attitude representation and the corresponding rotational kinematics can be referred to the work [16], [17].

\section{Dynamic Equations}

A variety of dynamic models for UAVs appear in the literature. Basically, the equations of motion are derived by implementing Newton-Euler formulas that deal with vector summations of all forces and moments applied to the aircraft relative to a reference frame. However, for practical control system design problems, it is very subtle to select appropriate frames and establish the motion model of UAVs. One should take into consideration simultaneously the characteristics of a specific aircraft, navigation accuracy, performance specifications and/or a control algorithm. In what follows, several commonly used motion models based on NewtonEuler law are introduced.

Assumption 2: An aircraft can be viewed as a rigid body with constant mass. The geometric shape and internal mass distribution of the aircraft are symmetrical with respect to the vertical plane $O x z$, i.e., the product of inertia $I_{x y}=I_{z y}=0$.

1) Translational dynamics: The translational equations are straightforward by applying Newton's second law, and the acceleration of an aircraft is derived from the vector summation of various forces. In terms of motion relative to the Earth, the translational equations written in the frame $\mathcal{B}$ introduce the Coriolis and centripetal terms, shown as follows:

$$
\left\{\begin{array}{l}
m(\dot{u}+w q-v r)=F_{x}^{B} \\
m(\dot{v}+u r-w p)=F_{y}^{B} \\
m(\dot{w}+v p-u q)=F_{z}^{B}
\end{array}\right.
$$

where $m$ denotes the mass of the aircraft, $\boldsymbol{F}^{B}=\left[F_{x}^{B}, F_{y}^{B}, F_{z}^{B}\right]^{T}$ denotes the principal vector $\boldsymbol{F}$ acting on the aircraft projected in the frame $\mathcal{B}$.

Remark 4: The resultant equations in terms of components in the aircraft-body frame are well posed even when all components become zero, e.g. hovering motion or standing on the runway. Equations (3) are commonly used for general flight simulation [12].

For a fixed-wing aircraft, it is usually more convenient to describe the aircraft speed and the flight state in the wind reference frame $\mathcal{W}$. In particular, the aerodynamic forces identified from a wind tunnel test are generally expressed in the frame $\mathcal{W}$ :

$$
\left\{\begin{aligned}
m \dot{V}=F_{x}^{W} & \\
m V \dot{\beta}=F_{y}^{W} & +m V(p \sin \alpha-r \cos \alpha) \\
m V \cos \beta \dot{\alpha}= & F_{z}^{W}-m V(p \cos \alpha \sin \beta-q \cos \beta \\
& +r \sin \alpha \sin \beta)
\end{aligned}\right.
$$

where $V$ denotes the airspeed of the UAV, $\boldsymbol{F}^{W}=$ $\left[F_{x}^{W}, F_{y}^{W}, F_{z}^{W}\right]^{T}$ denotes the principal vector $\boldsymbol{F}$ projected in the frame $\mathcal{W}$.

In addition, the translational dynamics can be expressed in the flight-path frame $\mathcal{P}$ (also known as ballistic coordinate system) as follows:

$$
\left\{\begin{array}{l}
m \dot{V}=F_{x}^{P} \\
m V \cos \gamma \dot{\chi}=F_{y}^{P} \\
-m V \dot{\gamma}=F_{z}^{P}
\end{array}\right.
$$

where $V$ denotes the inertial speed of the UAV which is equal to the airspeed in the absence of wind, $\boldsymbol{F}^{P}=\left[F_{x}^{P}, F_{y}^{P}, F_{z}^{P}\right]^{T}$ 
denotes the principal vector $\boldsymbol{F}$ projected in the frame $\mathcal{P}$.

Furthermore, by relating the total angular velocity to the rates $\dot{\chi}, \dot{\gamma}, \dot{\beta}$, and $\dot{\alpha}$ yields $\dot{\mu}$ :

$$
\begin{aligned}
\dot{\mu}= & \frac{p \cos \alpha+r \sin \alpha}{\cos \beta}+\frac{F_{y}^{P} \sin \gamma}{m V \cos \gamma} \\
& +\left(\frac{F_{y}^{P} \sin \mu}{m V}-\frac{F_{z}^{P} \cos \mu}{m V}\right) \tan \beta
\end{aligned}
$$

where $p$ and $r$ denote the roll and the yaw rate of a UAV about the axes of the frame $\mathcal{B}$.

2) Rotational dynamics: With the center of mass as a reference point, the rotational dynamics of an aircraft is separated from the translational dynamics, shown as follows:

$$
\left\{\begin{array}{l}
I_{x} \dot{p}+\left(I_{z}-I_{y}\right) q r-I_{x z} p q-I_{x z} \dot{r}=M_{x}^{B} \\
\left(I_{x}-I_{z}\right) p r+I_{y} \dot{q}+I_{x z}\left(p^{2}-r^{2}\right)=M_{y}^{B} \\
-I_{x z} \dot{p}+\left(I_{y}-I_{x}\right) q r+I_{x z} q r+I_{z} \dot{r}=M_{z}^{B}
\end{array}\right.
$$

where $I_{x}, I_{y}$ and $I_{z}$ denote the moments of inertia, $I_{x z}$ denote the product of inertia, and $\boldsymbol{M}^{B}=\left[M_{x}^{B}, M_{y}^{B}, M_{z}^{B}\right]^{T}$ denotes the principal moment acting on the aircraft with respect to the center of mass projected in the frame $\mathcal{B}$.

Remark 5: It is worth mentioning that the moments of inertia and the products of inertia are fixed measured from the frame $\mathcal{B}$. The aerodynamic moments identified from a wind tunnel test are commonly expressed in the frame $\mathcal{W}$. Thus, the aircraft-body axis equations (7) are the best choice for attitude control design.

\section{Summary}

The motion equations derived in this section are established based on the basic kinematic and dynamic laws. However, they can be written in different forms or different reference frames. To analyze and design a flight control system based on a control-oriented model of a UAV, it is essential to choose appropriate coordinate systems to define and describe the relevant motion parameters. For instance, unmanned rotorcraft UAVs (e.g., helicopter, quadrotor) are very suitable for quasistatic flight with small attitudes [18]. Thus, it is preferable to use aircraft-body equations (3) and (7) for control and simulation [19]-[21]. However, for super-manoeuvrable aircraft, it is required to hold sideslip angle near zero in order to improve the manoeuvrability and reduce the impact of heat flow at high speed [22]. It is justified to select the angle of attack, sideslip angle and bank angle for motion control. Besides, as shown in [23], [24], the motion of a symmetric UAV (especially missile) about its velocity vector can be alternatively described by using body axis accelerations instead of forces. The dynamic equations for the body axis accelerations at the center of mass are given by

$$
\left\{\begin{array}{l}
\dot{V}=\left(a_{x} \cos \alpha+a_{y} \sin \alpha\right) \cos \beta+a_{y} \sin \beta \\
\dot{\alpha}=q-(r \sin \alpha+p \cos \alpha) \tan \beta-\frac{a_{x} \sin \alpha-a_{z} \cos \alpha}{V \cos \beta} \\
\dot{\beta}=p \sin \alpha-r \cos \alpha-\frac{\left(a_{x} \cos \alpha+a_{z} \sin \alpha\right) \sin \beta-a_{y} \cos \beta}{V}
\end{array}\right.
$$

and the bank angle dynamics is described by

$$
\dot{\mu}=\frac{p \cos \alpha+r \sin \alpha}{\cos \beta}+\frac{\left(a_{x} \sin \alpha-a_{z} \cos \alpha\right) \tan \beta}{V}
$$

where $a_{x}, a_{y}$, and $a_{z}$ are body axis accelerations at the center of mass. Indeed, the acceleration dynamics (8) and (9) are equivalent to force dynamics (4) or (5) and (6), respectively. However, in addition to be capable of high angle of attack flight, the autopilot system design based on (8) and (9) may achieve higher control accuracy in the sense that the navigation system can provide accelerations and angular velocities in real-time, thus avoiding off-line calculation of external forces.

In brief, motion equations for UAVs can be various. One should balance the ease of representation of variables and control tasks of specific aircraft.

\section{Flight Control Methods}

This section gives a survey of commonly used flight control methods for UAVs, including classical control methods and advanced control designs based on exact models or uncertain models. Table I lists a summary of these methods. Specifically, linear/linearized or exact nonlinear model based control designs are classified into the category of classical control, while advanced control concerns about perturbed or uncertain model in this survey.

\section{A. Classical Control}

In Section III, several motion equations are given based on different representations or reference frames. Without loss of generality, the aircraft model can be formulated as the following affine nonlinear system:

$$
\left\{\begin{array}{l}
\dot{\boldsymbol{X}}=f(\boldsymbol{X}, \boldsymbol{U}) \\
\boldsymbol{Y}=h(\boldsymbol{X})
\end{array}\right.
$$

where $\boldsymbol{X} \in \mathbb{R}^{n}, \boldsymbol{U} \in \mathbb{R}^{m}$ and $\boldsymbol{Y} \in \mathbb{R}^{s}$ are the system state, input and output, respectively, $f: \mathbb{R}^{n} \times \mathbb{R}^{m} \rightarrow \mathbb{R}^{n}$ and $h: \mathbb{R}^{n} \rightarrow \mathbb{R}^{s}$ are nonlinear functions.

1) Linearized control methods: Traditional flight control law design is based on small perturbation theory. Specifically, linearize (10) at an equilibrium point $\left(\boldsymbol{X}_{e}, \boldsymbol{U}_{e}\right)$ or trimmed motion (usually satisfying $f\left(\boldsymbol{X}_{e}, \boldsymbol{U}_{e}\right)=0$ ) by invoking the Taylor series expansion. With a small perturbation, the highorder terms in the Taylor series expansion can be safely ignored, which leads to a linear time-invariant model which governs the system's dynamics at the trimmed point or motion:

$$
\dot{\tilde{X}}=A \tilde{\boldsymbol{X}}+B \tilde{\boldsymbol{U}}
$$

where $\quad \tilde{\boldsymbol{X}} \triangleq \boldsymbol{X}-\boldsymbol{X}_{e}, \quad \tilde{\boldsymbol{U}} \triangleq \boldsymbol{U}-\boldsymbol{U}_{e}, \quad A \triangleq \partial f\left(\boldsymbol{X}_{e}, \boldsymbol{U}_{e}\right) / \partial \boldsymbol{X}, \quad$ and $B \triangleq \partial f\left(\boldsymbol{X}_{e}, \boldsymbol{U}_{e}\right) / \partial \boldsymbol{U}$.

Based on the linearized model (11), the UAV dynamics can be decoupled into a vertical and a lateral motion [25]. In early flight control system design, eigenvalue assignment is applied successfully, by which the desired closed-loop performance can be guaranteed. For example, an eigenvalue assignment method is used to design the stability augmentation control 
TABLE I

CONTROL METHODS FOR UAVS

\begin{tabular}{|c|c|c|c|}
\hline Main categories & Control methods & Ref. & Characteristics \\
\hline \multirow{9}{*}{ Classical Control } & $\begin{array}{l}\text { Small perturbation linearization }+ \text { Eigenstructure } \\
\text { assignment approach }\end{array}$ & {$[26],[29]$} & $\begin{array}{l}\text { Guarantees local stability with small working } \\
\text { range, time-consuming }\end{array}$ \\
\hline & Gain scheduling approach & {$[30],[34]$} & $\begin{array}{l}\text { Guarantees local stability with large working } \\
\text { range, time-consuming }\end{array}$ \\
\hline & $\begin{array}{l}\text { Linear parameter varying (LPV) system + Robust } \\
H_{\infty} \text { control }\end{array}$ & {$[38]$} & $\begin{array}{l}\text { Guarantees global stability with certain } \\
\text { robustness, time-consuming }\end{array}$ \\
\hline & Linear quadratic regulator (LQR) & {$[39],[40]$} & $\begin{array}{l}\text { An optimal control approach, engineering } \\
\text { friendly }\end{array}$ \\
\hline & Nonlinear dynamic inversion (NDI) control & {$[22],[42]$} & Applicable to fully-actuated systems \\
\hline & Inner-outer loop structure + NDI & {$[22],[42]$} & $\begin{array}{l}\text { Based on a two timescale assumption, } \\
\text { applicable to under-actuated systems }\end{array}$ \\
\hline & Inner-outer loop structure + NDI + Command filter & {$[44]$} & $\begin{array}{l}\text { Alleviate the dependence of the closed-loop } \\
\text { performance on two timescale assumption }\end{array}$ \\
\hline & Backstepping control & {$[46],[47]$} & $\begin{array}{l}\text { Applicable to systems in strict feedback form, } \\
\text { explosion of derivatives }\end{array}$ \\
\hline & Command filtered backstepping control & {$[49]$} & Overcome the explosion of derivatives \\
\hline \multirow{8}{*}{$\begin{array}{c}\text { Robust and } \\
\text { Adaptive } \\
\text { Control }\end{array}$} & $\mu$-synthesis + Dynamic inversion (DI) & {$[50]$} & Guarantees the robustness of the outer loop \\
\hline & Adaptive NDI & {$[51]$} & $\begin{array}{l}\text { Guarantees the closed-loop adaptation, } \\
\text { unpredictable transient performance }\end{array}$ \\
\hline & Backstepping + Sliding mode control & {$[52]$} & $\begin{array}{l}\text { Guarantees the insensitivity to external } \\
\text { disturbances, chattering phenomenon }\end{array}$ \\
\hline & Adaptive backstepping & {$[53]$} & $\begin{array}{l}\text { Explosion of derivatives, unpredictable } \\
\text { transient performance }\end{array}$ \\
\hline & Command filtered adaptive backstepping & {$[55]$} & $\begin{array}{l}\text { Avoids explosion of the derivatives, } \\
\text { unpredictable transient performance }\end{array}$ \\
\hline & $\mathcal{L}_{1}$ adaptive control & {$[58],[59],[60]$} & $\begin{array}{l}\text { Admit fast adaptation with robustness and } \\
\text { guaranteed transient performance }\end{array}$ \\
\hline & Sophisticated cascaded control & {$[61],[62]$} & A saturated high gain design \\
\hline & Sensor-based incremental design + NDI & {$[63],[64]$} & $\begin{array}{l}\text { Address the robustness against unsmatched } \\
\text { disturbances }\end{array}$ \\
\hline \multirow{4}{*}{$\begin{array}{c}\text { Disturbance } \\
\text { Rejection } \\
\text { Control }\end{array}$} & Traditional disturbance observer-based control & {$[77],[76],[79]$} & Requires decoupling and linearization \\
\hline & Nonlinear disturbance observer-based control & {$[72],[81],[82],[73],[84]$} & Without decoupling and linearization \\
\hline & Extended state observer-based control & {$[85],[82],[86],[87]$} & Without requiring an accurate UAV model \\
\hline & Sliding mode disturbance observer & {$[88],[89],[74],[87]$} & Guarantees finite-time stability \\
\hline
\end{tabular}

system for a helicopter in hover flight [26]. A drawback of the eigenvalue assignment method is that the derived feedback control gain $\boldsymbol{K}$ is non-unique due to the freedom in assigning the associated eigenvectors. It is shown in [27] that if and only if the closed-loop eigenvalues are distinct, the gain $\boldsymbol{K}$ exists, which yields prescribed eigenvalues and eigenvectors. For linear multivariable systems, a general solution of eigenstructure assignment is provided in [28]. An application of the eigenstructure assignment approach is presented in [29] for flight control design. It should be emphasized that each element of the closed-loop eigenvector may not be completely independent which leads to the restriction on the application of the eigenstructure assignment. To be specific, for any closed-loop eigenvalue $\lambda_{i}^{d}, \quad i=1,2, \ldots, n$, the associated eigenvector $\boldsymbol{v}_{i}$ should satisfy $\boldsymbol{v}_{i}=\left(\lambda_{i}^{d} \boldsymbol{I}-\boldsymbol{A}\right)^{-1} \boldsymbol{B}\left(-\boldsymbol{K} \boldsymbol{v}_{i}\right)$. This implies that the closed-loop eigenvector $\boldsymbol{v}_{i}$ belongs to $\mathrm{m}$ dimensional subspace of $\boldsymbol{L}_{i} \triangleq\left(\lambda_{i}^{d} \boldsymbol{I}-\boldsymbol{A}\right)^{-1} \boldsymbol{B}$. For UAV systems, $m<n$ implies that $n$ elements of $\boldsymbol{v}_{i}$ cannot be prescribed arbitrarily.

These classical synthesis methods are able to achieve satisfactory performance only in a small range of trimmed point where the linearized model (11) works. For a large flight envelope, the closed-loop performance may deteriorate significantly and even the closed-loop stability cannot be guaranteed. Gain scheduling [30] was once widely used in flight control systems, which involves interpolating the linear control law design at intermediate operating conditions. Based on the monitored operating condition, the gains in the linear controller are scheduled accordingly. The principles for gain scheduling include gain interpolation, neural network [31], genetic algorithm [32], and fuzzy logic [33]. Take the work [34] for instance, a fuzzy gain scheduled controller is presented for an unmanned helicopter to achieve attitude stabilization within large ranges. Two prominent gain scheduling guidelines [35] can be raised: i) the scheduling variable should capture the plant's nonlinearities and ii) the scheduling variable should vary slowly. Hence, gain scheduling methodology cannot indeed guarantee global stability and robustness.

To remove these limitations due to gain scheduling, a theory for linear parameter varying (LPV) systems [36] is developed. The state space description of an LPV system can be written as follows: 


$$
\left\{\begin{array}{l}
\dot{\boldsymbol{X}}=\boldsymbol{A}(\varpi(t)) \boldsymbol{X}+\boldsymbol{B}(\varpi(t)) \boldsymbol{U} \\
\dot{\boldsymbol{Y}}=\boldsymbol{C}(\varpi(t)) \boldsymbol{X}+\boldsymbol{D}(\varpi(t)) \boldsymbol{U}
\end{array}\right.
$$

where the state-space matrices depend affinely on a timevarying real vector $\varpi(t)$. From a practical standpoint, LPV system (12) can be viewed as a linear time-invariant system with an uncertain time-varying parameter $\varpi(t)$, or a system derived by linearizing a nonlinear system along parameter trajectory $\varpi(t)$. In [37], the self-scheduled $H_{\infty}$ control is considered for LPV systems with an assumption that $\varpi(t)$ varies in a polytope $\Omega$ of vertices $\varpi_{1}, \varpi_{2}, \ldots, \varpi_{r}$. The statespace matrices evolve in a polytope of matrices:

$$
\Omega:=\sum_{i=1}^{r} a_{i} \Omega_{i}=\sum_{i=1}^{r} a_{i}\left[\begin{array}{ll}
A\left(\varpi_{i}\right) & B\left(\varpi_{i}\right) \\
C\left(\varpi_{i}\right) & D\left(\varpi_{i}\right)
\end{array}\right]
$$

where $a_{i}>0$ and $\sum_{i=1}^{r} a_{i}=1$. To this end, a robust $H_{\infty}$ control problem for polytopic LPV systems can be formulated and the resulting controller is therefore automatically gain-scheduled along the parameter trajectories. An application of LPV synthesis is presented in [38] for a missile and the resulting controller ensures the performance specifications over a wide operating envelope. In general, however, gain scheduling methodologies are time-consuming.

Linear quadratic regulator (LQR) reduces the amount of work done by control engineers to optimize a controller. In early flight control system designs, LQR is commonly used and its successful applications include F-8C and X-29 prototypes. Essentially, LQR is an optimal control approach with a cost function described by a quadratic form. In [39], an application of $\mathrm{LQR}$ for the missile guidance is shown and the flight test results are also provided. In [40], a normal LQR controller is developed for a helicopter to achieve hovering flight, which combines an active model enhancement technique by means of the unscented-Kalman-filter (UKF) estimation.

2) Nonlinear control methods: It is shown in Section III that the motion equations of an aircraft present a highly nonlinear and coupling dynamics. Linearized model-based synthesis techniques encounter difficulties in guaranteeing stability and robustness in a full flight envelope. In addition to the performance limitation, flight missions achieved by classical control are relatively simple. As a result, for modern UAVs, nonlinear control techniques are more appealing and competitive in improving flight performance and reducing workload for flight engineers.

Linearization feedback or nonlinear dynamic inversion [41] technique provides a straightforward means of deriving control laws for nonlinear systems. In particular, the unwanted terms in the equations of motion are cancelled by the input using negative feedback of these terms. For example, given a set of outputs $\boldsymbol{Y}=\left[y_{1}, y_{2}, \cdots, y_{s}\right]^{T}$ and their relative degrees are denoted by $r_{i}, i=1,2, \ldots, s$, respectively, by successive differentiation, one obtains

$$
\left[\begin{array}{c}
y_{1}^{\left(r_{1}\right)} \\
\vdots \\
y_{s}^{\left(r_{s}\right)}
\end{array}\right]=\overline{\boldsymbol{A}}(\boldsymbol{X})+\overline{\boldsymbol{B}}(\boldsymbol{X}) \boldsymbol{U}
$$

Provided that $\overline{\boldsymbol{B}}(\boldsymbol{X})$ is nonsingular for $\forall \boldsymbol{X} \in \mathbb{R}^{n}$, the exact input-output linearization feedback control law can be proposed as

$$
\boldsymbol{U}=\overline{\boldsymbol{B}}^{-1}(\boldsymbol{X})(\overline{\boldsymbol{U}}-\overline{\boldsymbol{A}}(\boldsymbol{X}))
$$

where $\bar{U}$ is a new input to be designed. With (14), the resultant system (13) is a linear system and various wellknown control techniques can be applied. Classical paradigms of how to apply the nonlinear dynamic inversion in flight control design are set up in some pioneer works (e.g., [22], [42]). However, the dynamic inversion approach requires that a system has at least as many inputs as controlled states. In aircraft control systems, this is not generally the case since many UAVs are under-actuated, such as helicopter, fixedwing aircraft. Hence, the conventional flight control problem is formulated as a two timescale problem, which, in turn, forms an inner-outer loop control structure to solve the underactuation problem. However, stability analysis for such a control structure could be challenging. In [43], the stability for a missile system with a dynamic inversion controller is proved and a minimum gain for the inner-loop controller is derived. The closed-loop stability relies on an assumption that the frequency of the desired dynamics in the inner-loop inversion is sufficiently large. However, the large inner-loop gain easily excites unmodeled dynamics and saturates the inputs, which imposes a restriction on the application of nonlinear dynamic inversion in practical flight control designs. To eliminate the two timescale assumption, a solution of using a command filter is proposed in [44], which produces the derivatives of the command signal required in the inner-loop control of a quadrotor. This removes the requirement of a large inner-loop gain, but a rigorous stability proof for the overall inner-outer loop structure cannot be given explicitly.

The backstepping technique [45] is frequently employed in the design of a flight controller for various UAVs, mainly due to the fact that the generic equations of motion (including the translation and the rotation) are in a strict feedback form:

$$
\left\{\begin{array}{l}
\dot{\boldsymbol{X}}_{1}=f_{1}\left(\boldsymbol{X}_{1}\right) \boldsymbol{X}_{2} \\
\dot{\boldsymbol{X}}_{2}=f_{2}\left(\boldsymbol{X}_{1}, \boldsymbol{X}_{2}\right)+g_{2}\left(\boldsymbol{X}_{1}, \boldsymbol{X}_{2}\right) \boldsymbol{U}_{1}
\end{array}\right.
$$

where $\boldsymbol{X}_{1}$ and $\boldsymbol{X}_{2}$ denote the position (Euler angle) and the velocity (angular velocity), respectively, and $\boldsymbol{U}_{1}$ denotes the control input. An example of applying backstepping for UAV hovering flight can be found in [46], which is based on a generic UAV model valid for quasi-stationary conditions, and the applicability for hover stabilization of an airship UAV is also shown. In [47], the backstepping based control design is presented for autonomous landing of a rotary-wing UAV and only a two-step procedure is required to obtain the control inputs thanks to the inner-outer loop strategy. However, the recursive backstepping design requires the analytic derivatives of virtual control variables, which leads to a complex control structure, especially for high-order systems. Compared to standard backstepping, command filtered backstepping [48] integrates command filters in each recursive step which not only eliminate the tedious analytic derivative computation of virtual control variables, but also impose physical constraints 
on them if necessary. For example, in [49], the command filtered backstepping design is presented to the longitudinal control of an aircraft subject to magnitude, rate, and bandwidth constraints on the aircraft states and the actuator signals. Although the complexity arising due to the explosion of derivatives of virtual control variables in the standard backstepping can be greatly alleviated, high-frequency noises pass through these command filters quite easily. Thus, control accuracy may deteriorate as the bandwidth of command filters increases in flight control design.

\section{B. Robust and Adaptive Control}

The exact model-based control techniques discussed in Subsection IV-A are usually very sensitive to any variation of model parameters, thus lacking robustness and adaptation. In particular, exact dynamics of an aerial vehicle can hardly be established and some external disturbances cannot be measured either. This requires the development of more advanced control methodologies to handle model uncertainties as well as unknown disturbances.

To account for system uncertainties and exogenous disturbances, the state-space equation of the aircraft can be rewritten as

$$
\dot{\boldsymbol{X}}=f(\boldsymbol{X}, \boldsymbol{W}, \boldsymbol{U})=\bar{f}(\boldsymbol{X})+\bar{g}(\boldsymbol{X}) \boldsymbol{U}+\boldsymbol{\Delta}(\boldsymbol{X}, \boldsymbol{W})
$$

where $\bar{f}(\boldsymbol{X})$ and $\bar{g}(\boldsymbol{X})$ are the nominal model of the aircraft, i.e., the known modeled dynamics, $\boldsymbol{W}$ denotes exogenous disturbances or states, and $\boldsymbol{\Delta}$ represents the unmodeled dynamics. In most of the existing literature, a baseline controller, applied for the nominal dynamics $\dot{\boldsymbol{X}}=\bar{f}(\boldsymbol{X})+$ $\bar{g}(\boldsymbol{X}) \boldsymbol{U}$, is augmented with an additional design that deals with the term $\Delta$.

Robust control can be seen from early work such as [50], where inner-loop dynamic inversion equalizes plant dynamics across the flight envelope and outer-loop $\mu$-synthesis around the equalized plant is used to guarantee the performance and robustness. The adaptive nonlinear dynamic inversion approach becomes more popular (e.g., [51]), where adaptive control is used to counteract the dynamic inversion error. Using formulation (15), the dynamic inversion error can be written as $\boldsymbol{\Delta}=f_{2}-g_{2} \bar{g}_{2}^{-1} \bar{f}_{2}$, where $\bar{f}_{2}$ and $\bar{g}_{2}$ denote the nominal functions corresponding to $f_{1}$ and $f_{2}$ in (15). In [52], a backstepping controller mixed with sliding mode control is proposed for a quadrotor helicopter. Specifically, the backstepping controller can be viewed as a baseline controller and the sliding mode control is used to compensate the effects of model uncertainties and external disturbances. Another backstepping based adaptive tracking controller is developed for a quadrotor UAV subject to mass uncertainty [53].

Similar to standard backstepping, the adaptive version also suffers from the problem of the explosion of complexity arising from the differentiation of intermediate virtual control. Command filtered adaptive backstepping [54] provides an implementation approach for adaptive backstepping control by introducing filters to generate compensating signals for adaptive parameter estimation. In [55], the command filtered adaptive backstepping design is presented to solve the trajectory tracking problem for a quadrotor with unknown aerodynamic drag coefficients and external disturbances. Note that the adaptation rate relates closely to the closed-loop performance and stability. In other words, the closed-loop transient performance is unpredictable and the high adaptation rate will deteriorate the closed-loop stability [56]. Notably, $\mathcal{L}_{1}$ adaptive control theory [57] allows for fast adaptation with guaranteed robustness. The key properties of $\mathcal{L}_{1}$ adaptive controller are validated in several benchmark flight tests as shown in [58], [59]. In particular, the $\mathcal{L}_{1}$ adaptive backstepping design is presented for the translational dynamics of a multirotor UAV, using a piecewise constant adaptation law to estimate the lumped unknown term $\Delta$ in the presence of fast adaptation [60].

Robust controllers are also designed for VTOL UAVs. In [61], a sophisticated cascaded control structure is developed for a small helicopter by combining feed-forward control actions and high-gain controller with nested saturation function. It is proved in the experiments that the control design is able to robustly track the trajectory tracking with respect to various system uncertainties. A similar design approach is also applied to a ducted-fan UAV with solid experimental results [62].

Another interesting control design paradigm is the sensorbased incremental design combined with NDI [63] or other alternatives. It uses sensor measurements to replace part of the system model information when dealing with model mismatch uncertainties. This control scheme is demonstrated in flight tests with promising results [64]. It should be noted that there are many other control approaches also incorporating an observer to improve the robustness and control performance [65], [66].

\section{Disturbance Rejection Control}

Disturbance rejection control aims to actively compensate or attenuate the influence of external disturbances acting on the UAV dynamics, so as to improve the flight performance. The basic principle of disturbance rejection control is first to design an observer to estimate the lumped disturbance term $\Delta$, as in (16), and then to exploit such an estimate $\hat{\Delta}$ in control design to eliminate its influences. There are several observer design that can be applied for UAV fight control, including disturbance observers [67], extended state observers [68] and sliding mode observers [69], [70]. A comprehensive review on disturbance rejection control can be found in [71], with a focus on disturbance observer based control techniques.

As indicated in [71], the disturbance observer based design paradigm exhibits several advantages when it is applied to UAV flight control design. Such a technique can be regarded as a "patch" function to the existing flight controllers, which offers flexibility in control integration. In terms of UAV control, disturbance observer based control is integrated with nonlinear dynamic inversion [72], backstepping [73], sliding mode control [74], [75], and $H_{\infty}$ [76], [77]. Another feature of disturbance observer based control is the capability of recovering the nominal control performance. The baseline feedback controller can focus on delivering the tracking performance and stability, while the disturbance rejection and robustness against uncertainties can be left to the 
compensation loop. This means in the absence of disturbances/uncertainties, the nominal control performance of a UAV thus can be retained.

A traditional disturbance observer in the frequency domain is proposed [78], which has two key steps: the inverse of a nominal plant model and the design of a low pass filter. Since this disturbance observer is mainly designed for single-inputsingle-output linear time-invariant systems, it is usually used to deal with decoupled and linearized dynamics of a UAV. In [77], linearized models around the hovering condition of a tail-sitter are obtained via a frequency sweep method and used in disturbance observer design. Similarly, in [76], a disturbance observer is applied to inner loop control of a quadrotor, but the nominal model used in disturbance observer design is an error dynamics obtained through feedback linearization. It should be noted that the $H_{\infty}$ synthesis is employed to balance the robustness and the disturbance rejection performance [78], [76]. For more complicated UAV systems, such as a fully actuated thruster-tilting multicopter [79], a more sophisticated decoupling and simplification process will be required.

Compared to the disturbance observer in the frequency domain, a more flexible and effective formulation is a nonlinear disturbance observer proposed in [80]. This design allows the nonlinear model to be directly exploited in control design rather than being treated as disturbances, which also means that decoupling and linearization may not be necessary in flight control design. Such a scheme is deployed on a small helicopter to perform an accurate pirouette maneuver by addressing the coupling effect between the lateral and longitudinal dynamics [72] and on a quadrotor to improve the tracking performance under the ground effect [81]. Some variations of this disturbance observer structure have been developed in recent years to deal with many other difficulties. One direction is to incorporate a more comprehensive model to represent different types of disturbances. For example, exogenous disturbances, which include periodic disturbances caused by a slung payload or composed with different frequency harmonics, are addressed in [82] and [83], respectively. It should be noted that the original nonlinear disturbance observer design is a reduced-order observer, which means that all the system states should be available to the observer. Since the onboard sensors may be limited to small UAVs, the nonlinear disturbance observer has been extended to deal with partially measurable system states, which are often raised from actuator dynamics of the UAV. Examples include the disturbance observer design to accommodate unmeasurable flapping dynamics of an unmanned helicopter [73] and unknown actuator states of the control surfaces on a fixed-wing UAV [84].

Another popular disturbance rejection approach is based on an extended state observer (ESO). The traditional ESO design does not require a specific model of the system. Instead, the system dynamics needs to be cast into a chain of integrators based on the relative degrees [68]. This may be convenient in practice since it is not always easy to obtain an accurate UAV model. However, abandoning the knowledge of a system model may induce high frequency fluctuations in the control signal, especially when the system order is high. Therefore, ESO based controllers are normally used for low-order subsystems such as individual attitude/translational dynamics in the cascaded control structure to replace traditional PID controllers [85]. More recent design techniques such as feedback linearization are used to derive the linear models of quadrotors for ESO design [82], [86]), where the model information is partially incorporated. The nonlinear ESO design for quadrotors using a detailed angular velocity model is presented in [87] with promising experimental results against wind gusts.

A sliding mode disturbance observer is a powerful tool in flight control design. It does not rely on the complete knowledge of the disturbance model. Instead, with just information of disturbance bounds, the simple low-order observer design can be realized, which can also estimate the derivatives of disturbances. This feature is very useful as most of the disturbances on UAVs are difficult to model. After initially demonstrated on a reusable launch vehicle [88], an sliding mode disturbance observer is used on quadrotors [89], [74] and fixed-wing UAVs [87] for various applications. The sliding mode disturbance observer design also offers a framework to establish theoretical properties such as finitetime stability and incorporate high-order disturbance models. However, the benefit of using the high-order disturbance model still requires further evidence from flight tests, as most of the relevant studies still rely on numerical simulations.

\section{TASK SPECIFIC CONTROL DESIGN}

The ultimate goal of flight control design is to enable UAVs to perform specific tasks automatically. Some task requirements can be formulated or converted to a standard control problem, thus can be solved by using above mentioned control methods. The most common UAV tasks would be trajectory tracking and path following functions, which usually serve as the basis of more complicated flight missions, such as formation flight and drone racing. The main solution depends on the definition of a trajectory- or path-dependent error space to express the dynamic model of a UAV in such tasks. Then, various standard control methods can be applied to solve the trajectory tracking or path-following problem, such as gain scheduling [90], feedback linearization [91], sliding mode control [92], $\mathcal{L}_{1}$ adaptive control [58].

\section{A. Trajectory Tracking Problem}

Definition 5 (Trajectory tracking problem): Given a sufficiently smooth desired trajectory $\boldsymbol{p}_{d}^{E}(t)$ with bounded derivatives $\dot{\boldsymbol{p}}_{d}^{E}$ and $\ddot{\boldsymbol{p}}_{d}^{E}$, design a controller such that all the closed-loop signals are bounded and the trajectory tracking error $\left\|\boldsymbol{p}^{E}(t)-\boldsymbol{p}_{d}^{E}(t)\right\|$ converges to an arbitrarily small neighbourhood of the origin.

Trajectory tracking problems are concerned with controller design that force a vehicle to reach and follow a geometric path with an associated timing law. In this sense, the trajectory tracking based control system design is quite suitable for high maneuverability [93] and time-critical missions [94].

To solve the trajectory tracking problem, the difficulty highly relies on the configuration of an aircraft. For fully- 
actuated systems, the trajectory tracking problem has been well understood. However, many UAVs, including spacecraft, aircraft, helicopters and missiles, are typical under-actuated systems. The trajectory tracking problem for under-actuated UAVs is especially challenging because these systems are in general not fully feedback linearizable, and therefore standard tools, such as feedback linearization and backstepping, are not straightforwardly applicable [95].

The classical trajectory tracking control of under-actuated systems uses decoupling of a multi-variable model to steer the same number of degrees of freedom as the number of available control inputs. Taking a helicopter for example, it is common to separate the control problem into an inner-loop that stabilizes the attitudes and an outer-loop that controls the trajectories of the vehicle [96]. It should be emphasized that the desired trajectory does not need to be a trimming one and can be any sufficiently smooth bounded curve parameterized by time. This provides UAVs with more flexible task-oriented control design.

\section{B. Path-Following Problem}

Definition 6 (Path-following problem): Let $\boldsymbol{p}_{d}^{E}(\xi)$ be a desired path, parameterized by $\xi \in \mathbb{R}$, and $\boldsymbol{V}_{d}(\xi)$ be the desired velocity. For a given sufficiently smooth path $\boldsymbol{p}_{d}^{E}(\xi)$ with bounded derivatives with respect to $\xi$, design a controller such that all the closed-loop signals are bounded and the pathfollowing error $\left\|\boldsymbol{p}^{E}(t)-\boldsymbol{p}_{d}^{E}(\xi(t))\right\|$ converges to an arbitrarily small neighbourhood of the origin. In addition, the real velocity $\boldsymbol{V}(t)$ along the given path $\boldsymbol{p}_{d}^{E}(\xi(t))$ converges to the desired velocity $\boldsymbol{V}_{d}(\xi(t))$ in a desired manner.

Different from the trajectory tracking control problem, the path-following control problem is decoupled into a geometric path-following problem and a speed-assignment problem. The geometric task is to stabilize a family of trajectories with the same geometric pattern but with diversely admissible speed profiles, called path following manifold [91], [98]. The remaining speed assignment, which is usually given as a timing law, determines the diversity. In some non-minimum phase systems, the timing law for $\xi(t)$ can be well designed to stabilize the unstable zero dynamics [97]. Due to the extra timing law $\dot{\xi}(t)$, path-following control is more flexible than trajectory tracking control. In this sense, path-following control is quite suitable for some UAVs with physical speed restrictions, such as fixed-wing UAVs [99], [100], rotary-wing UAVs [101] and airships [102], [103], and for some specific missions without temporal requirements.

One of the typical missions is target tracking which covers many military applications, such as adversarial target observation and friendly convoy protection. In [104], a visionbased target tracking guidance algorithm is proposed with an image motion estimation system. The guidance law prescribes the UAV flying perpendicular to the line of sight vector and regulates the speed to maintain a constant range between two vehicles. Lyapunov guidance vector fields are developed for autonomous coordination of multiple unmanned aircraft [105], which yields feasible and globally stable paths with guaranteed target stand-off distance bounds. The loitering tracking strategy is further improved and extended in [106] and [107] by speeding the convergence process and imposing a curvature limitation, respectively.

\section{Future Challenges AND Research Directions}

As the principles of UAV flight control have been well understood over last two decades, commercial efforts and open-source community have delivered a good level of maturity for flying common UAV platforms in open space. The growth in technology readiness and reliability of UAVs has fueled the interest in deploying UAV platforms in wider and more challenging scenarios, which, in turn, poses higher requirements on flight control system design.

\section{A. Challenging Issues}

Loosely speaking, many of the remaining technical challenges may arise from two aspects, i.e., unknown/uncertain dynamics and external operating environments.

- Unknown/uncertain dynamics: Some UAV platforms, especially those with unconventional configurations, such as over-actuated multirotors [108], hybrid UAVs [109] and bioinspired UAVs [110], may suffer from complex and poorly unknown dynamics. The same issue may also be encountered when pushing UAVs to their dynamics limits, such as in drone racing and operating under partial faults. Another example is aerial transportation, including suspending the payload [111] or grasping the payload [112], where the influence of the payload could be difficult to capture. In such situations, traditional modeling means, such as wind tunnel testing and system identification may be too expensive to run or inadequate to provide satisfactory results within a desired time frame.

- Unknown/uncertain external operating environments: Many applications may require UAVs to be operated in an unknown, or partially known environment, which could be dynamically changing at the same time. Such an application may also include UAVs flying in close proximity to objects in the environment (e.g., for infrastructure inspection) or having physical interactions with the environment (e.g., aerial manipulators). The flight control design thus needs to take into account the performance or imperfection of the sensing and perception systems in a more systematical way.

It should be noted that the technical challenges from those two areas are often coupled. Physical interactions with operational environments usually exert external forces and moments on UAV dynamics, which are difficult to capture or model. Flying in unknown and dynamic environments may require the UAVs to take aggressive manoeuvres to avoid obstacles that can only be detected in a close range. To this end, the design and implementation of sophisticated control techniques that can work in conjunction with advanced estimation and perception algorithms would be a focal point of future research on UAV flight control.

\section{B. Continuing Research}

In recognition of those challenges in UAV flight control design, there are a number of emergency control design methods that have already shown the potential for addressing 
some of the issues.

Traditional control design methods to a large extent leverage the knowledge of the system dynamics in the form of a system model but could be less effective when incorporating knowledge of or influence from external environments (which are often regarded simply as disturbances or uncertainties). To this end, learning-based control methods, also known as intelligent control, may offer an alternative way of dealing with unknown dynamics [113], challenging environments [114] and even intelligent actuators [115] involved in UAV operations. Note in recent years that the confluence of big data and machine learning is steering a paradigm shift in the analysis and understanding of dynamical systems in UAV engineering. One of the most noteworthy is the so-called Koopman operator theory [116] which opens a path to identify intrinsic coordinate systems to represent nonlinear dynamics in a linear framework. It could be expected that obtaining linear representations of strongly nonlinear systems has the potential to revolutionize our ability to predict and control complex UAV systems in large-scale and dynamic changing environments [117]. Hence, the integration of data driven and learning based techniques with dynamical systems theory opens up a new opportunity to tackle previously unattainable challenge problems in modeling and control of UAVs without detailed system knowledge.

Control with safety guarantees for UAVs is another important area to be investigated, and there are several daunting technical problems to tackle especially when UAVs are making their way into civilian applications. New control technologies must be safe and reliable enough for the general public to have confidence that nothing will go wrong. Control design with constraints in mind, such as using a barrier Lyapunov function [118], would be a useful solution to realize the position constrained trajectory for UAVs deployed in confined areas. In addition, the UAV operation in close proximity to structures and the terrain is more difficult when navigation information is not fully available (e.g., GPS dropouts) and collision avoidance is obligatory. This requires an integrated control system in terms of perception [119], state estimation [120], and obstacle avoidance algorithms [121]. It is even more challenging when navigating multiple UAVs safely and efficiently in a large-scale airspace with both static and dynamic obstacles under wind disturbances. Data-driven based control methods (e.g., [122]) may have a great potential to enable safe and efficient UAV operations in a large-scale dynamic civilian environment. However, accidents may happen when the training data is noisy or the training process does not fit the real-world scenarios [123] and rigorous stability proof is usually unaccessible [124]. To this end, continuing research is required to establish more formal verification and validation frameworks to guarantee the safety of emerging control methods for UAVs.

\section{CONCLUDING REMARKS}

As discussed in this survey, established model-based flight control methods have made a great success in delivering automatic flight functions for different types of UAVs. The maturity of basic flight control has in turn increased people's expectation of using them in more public domains and civilian airspace. On one hand, UAVs can offer enormous potential for applications in urban environment, ranging from infrastructure monitoring to traffic surveillance, emergency response and medical payload delivery. On the other hand, the complexity and diversity of the tasks and the uncertainties in the operation environments have posed more challenges on the autonomy, intelligence and safety features of UAV systems, which may be beyond the reach of existing modelbased flight control methods.

Recent advances in machine learning and data-driven methods, as well as emerging sensing and perception capabilities from robotics, show a promising technical pathway to build more autonomous and intelligent UAV systems by allowing them to learn from experience and perceive the environment. The combination of advanced sensing modalities and learning based algorithms for flight control design creates a series of technical, theoretical and safety challenges:

- Comprehensive frameworks, either through theoretical analysis or using numerical verification, need to be established to examine the system stability of the UAV when large level of uncertainties are introduced by advanced perception systems.

- New methodologies are required to verify and analyze the learning based algorithms beyond basic flight stability analysis, because real-world situations may breach the scenarios or datasets used in the training process.

- Suitable specifications for assessing the functionality of UAVs need to be developed to ensure safety and reliability in response to the diversity of the tasks in dynamic and unpredictable urban environment.

- Ethical and legal issues in relation to responsibility and liability of UAV operations should also be considered when designing more autonomous and intelligent functions.

To enable deployment of UAVs in civilian airspace, these grand challenges need to be addressed jointly by the UAV research and development community, but it is envisaged that a wider and profound impact will then be delivered on the society.

\section{REFERENCES}

[1] I. A. Raptis and K. P. Valavanis, Linear and Nonlinear Control of Small-Scale Unmanned Helicopters. New York: Springer Science, 2011.

[2] A. S. Saeed, A. B. Younes, C. Cai, and G. Cai, "A survey of hybrid unmanned aerial vehicles," Progress in Aerospace Sciences, vol.98, pp. 91-105, 2018.

[3] J. C. Doyle and G. Stein, "Multivariable feedback design: Concepts for a classical/modern synthesis," IEEE Trans. Automatic Control, vol.26, no. 1, pp. 4-16, 1981.

[4] A. N. Andry, E. Y. Shapiro, and J. C. Chung, "Eigenstructure assignment for linear systems," IEEE Trans. Aerospace and Electronic Systems, vol. AES-19, no. 5, pp. 711-729, 1983.

[5] D. Enns, D. Bugajski, R. Hendrick, and G. Sein, "Dynamic inversion: An evolving methodology for flight control design," Int. Journal of Control, vol. 59, no. 1, pp.71-91, 1994.

[6] S. Sastry and M. Bodson, Adaptive Control: Stability, Convergence and Robustness. New Jersey: Prentice-Hall, 1989. 
[7] B. D. O. Anderson, "Failures of adaptive control theory and their resolution," Communications in Information and Systems, vol. 5, no. 1, pp. 1-20, 2005.

[8] A. Datta and M. Ho, "On modifying model reference adaptive control schemes for performance improvement," IEEE Trans. Automatic Control, vol.39, no. 9, pp. 1977-1980, 1994.

[9] M. Arcak, M. Seron, J. Braslavsky, and V. Kokotović, "Robustification of backstepping against input unmodeled dynamics," IEEE Trans. Automatic Control, vol.45, no. 7, pp. 1358-1363, 2000.

[10] I. Kaminer, P. Khargonekar, and G. Robel, "Design of localizer capture and track modes for a lateral autopilot using $H_{\infty}$ synthesis," IEEE Control Systems Magazine, vol. 10, no. 4, pp. 13-21, 1990.

[11] R. W. Beard and T. W. McLain, Small Unmanned Aircraft: Theory and Practice. New Jersey: Princeton University Press, 2012.

[12] B. L. Stevens, L. F. L., and E. N. Johnson, Aircraft Control and Simulation. New Jersey: John Wiley \& Sons, 2016.

[13] R. J. Wallsgrove and M. R. Akella, "Globally stabilizing saturated attitude control in the presence of bounded unknown disturbances," Journal of Guidance, Control, and Dynamics, vol.28, no. 5, pp. 957-963, 2005.

[14] Tsiotras, "Stabilization and optimality results for the attitude control problem," Journal of Guidance, Control, and Dynamics, vol. 19, no. 4, pp. 772-779, 1996.

[15] R. M. Murray, Z. Li, and S. Sastry, A Mathematical Introduction to Robotic Manipulation. Boca Raton: CRC Press, 1994.

[16] J. T.-Y. Wen and K. Kreutz-Delgado, "The attitude control problem," IEEE Trans. Automatic Control, vol.36, no. 10, pp.1148-1162, 1991.

[17] M. D. Shuster, "A survey of attitude representation," Journal of the Astronautical Sciences, vol. 41, no. 4, pp.439-517, 1993.

[18] A. R. S. Bramwell, G. Done, and D. Balmford, Bramwell's Helicopter Dynamics. Oxford: Butterworth Heinemann, 2001.

[19] D. Cvetković, I. Kostić, C. Mitrović, and A. Bengin, "Mathematical models of helicopter flight dynamics," in Proc. 40th AIAA Aerospace Sciences Meeting \& Exhibit, AIAA 2002-0529, DOI: 10.2514/6.2002529, Reno, NV, 2002.

[20] B. Zhu and Z. Zuo, "Approximate analysis for main rotor flapping dynamics of a model-scaled helicopter with bell-hiller stabilizing bar in hovering and vertical flights," Nonlinear Dynamics, vol. 85, pp. 1705-1717, 2016.

[21] V. Gavrilets, B. Mettler, and E. Feron, "Nonlinear model for a smallsize acrobatic helicopter," in Proc. AIAA Guidance, Navigation, and Control Conf. and Exhibit, AIAA-2001-4333, DOI: 10.2514/6.2001-4333, Montrcal, Canada, 2001.

[22] S. A. Snell, D. F. Enns, and W. L. Garrard Jr., "Nonlinear inversion flight control for a supermaneuverable aircraft," Journal of Guidance, Control, and Dynamics, vol. 15, no. 4, pp.976-984, 1992.

[23] K. A. Wise and D. J. Broy, "Agile missile dynamics and control," Journal of Guidance, Control, and Dynamics, vol.21, no.3, pp. 441-449, 1998.

[24] M. B. McFarland and A. J. Calise, "Adaptive nonlinear control of agile antiair missiles using neural networks," IEEE Trans. Control Systems Technology, vol. 8, no. 5, pp. 749-756, 2000.

[25] R. C. Nelson, Flight Stability and Automatic Control. McGraw-hill, New York, 1998.

[26] K. L. Hicks and A. A. Rodriguez, "Decoupling compensation for the apache helicopter," in Proc. 35th IEEE Conf. on Decision and Control, Kobe, Japan, 1996, pp. 1551-1556.

[27] B. C. Moore, "On the flexibility offered by state feedback in multivariable systems beyond closed loop eigenvalue assignment," IEEE Trans. Automatic Control, vol.21, no. 5, pp.689-692, 1976

[28] M. M. Fahmy and J. O’Reilly, "On eigenstructure assignment in linear multivariable system," IEEE Trans. Automatic Control, vol.27, no.3, pp. 690-693, 1982.

[29] J. E. Piou and K. M. Sobel, "Application of gain scheduled eigenstructure assignment to flight control design," in Proc. the 1996 IEEE Int. Conf. on Control Applications, Dearborn, MI, 1996, pp.
$101-106$

[30] W. J. Rugh, "Analytical framework for gain scheduling," IEEE Control Systems Magazine, vol. 11, no. 1, pp. 79-84, 1991.

[31] X. Dong, Z. Xiong, and Q. Liu, "Gain scheduled model following control of flight control system based on neural network," in Proc. IEEE Int. Conf. on Neural Networks \& singnal Processing, Nanjing, China, 2003, pp. 301-305.

[32] G. J. Gray, Y. Li, D. J. Murray Smith, E. Ronco, and K. C. Sharman, "The application of genetic algorithms to gain-scheduling controller analysis and design," in Proc. IEE Conf. Publication, 1997, pp. 344-348.

[33] J. Varso and H. N. Koivo, "Fuzzy logic in gain scheduling of multivariable control," in Proc. the 3rd IEEE Conf. on Industrial Electronics and Application, 2008, pp. 1264-1269.

[34] B. Kadmiry, P. Bergsten, and D. Driankov, "Autonomous helicopter control using fuzzy gain scheduling," in Proc. the 2001 IEEE Int. Conf. on Robotics \& Automation, Seoul, Korea, 2001, pp. 2980-2985.

[35] J. S. Shamma and M. Athans, "Gain scheduling: potential hazards and possible remedies," IEEE Control Systems Magazine, vol.12, no.3, pp. 101-107, 1992

[36] J. F. Shamma and J. R. Cloutier, "A linear parameter-varying approach to gain scheduled missile autopilot design," in Proc. the American Control Conf., Chicago, IL, 1992, pp. 1317-1321.

[37] P. Apkarian, P. Gahinet, and G. Becker, "Self-scheduled control of linear parameter-varying systems," in Proc. the American Control Conf., Balffmore, Maryland, 1994, pp. 856-860.

[38] A. Hiret, G. Duc, and J. P. Friang, "Self-scheduled $H_{\infty}$ loop-shaping control of a missile," in Proc. the European Control Conf., Karlsruhe, Germany, 1999, pp. 1207-1212.

[39] E. J. Cramer and T. P. Lee, "Test flight of LQR missile guidance," Tech. Rep. AIAA-92-4532-CP, 1992.

[40] Z. Jiang, J. Han, Y. Wang, and Q. Song, "Enhanced LQR control for unmanned helicopter in hover," in Proc. 1st Int. Symposium on Systems and Control in Aerospace and Astronautics, Harbin, China, 2006, pp. 19-21.

[41] H. K. Khalil, Nonlinear Systems, 3rd ed. New Jersey: Princeton Hall, 2002.

[42] S. H. Lane and R. F. Stengel, "Flight control design using non-linear inverse dynamics," Automatica, vol.24, no.4, pp.471-483, 1988.

[43] C. Schumacher and P. Khargonekar, "Stability analysis of a missile control system with a dynamic inversion controller," Journal of Guidance, Control, and Dynamics, vol.21, no. 3, pp.508-515, 1998.

[44] Z. Zuo, "Trajectory tracking control design with command-filtered compensation for a quadrotor," IET Control Theory \& Applications, vol. 4, no. 11, pp. 2343-2355, 2010.

[45] M. Krstic, I. Kanellakopoulos, and P. Kokotovic, Nonlinear and Adaptive Control Design. New York: John Wiley \& Sons, 1995.

[46] J. R. Azinheira and A. Moutinho, "Hover control of an UAV with backstepping design including input saturations," IEEE Trans. Control Systems Technology, vol. 16, no. 3, pp. 517-526, 2008.

[47] B. Ahmed, H. R. Pota, and M. Garratt, "Flight control of a rotary wing UAV using backstepping," Int. Journal of Robust and Nonlinear Control Control, vol. 20, no. 6, pp. 639-658, 2010.

[48] J. Farrell, M. Polycarpou, and M. Sharma, "Command filtered backstepping," IEEE Trans. Automatic Control, vol.54, no.6, pp. 1391-1395, 2009.

[49] J. A. Farrell, M. Polycarpou, and M. Sharma, "Adaptive backstepping with magnitude, rate, and bandwidth constraints: Aircraft longitude control," in Proc. American Control Conf., Denver, Colorado, 2003, pp. 3898-3904.

[50] R. J. Adams, M. Xin, and S. S. Banda, "Robust flight control design using dynamic inversion and structured singular value synthesis," IEEE Trans. Control, Systems Technology, vol.1, no.2, pp.80-92, 1993.

[51] R. Hindman and W. M. Shell, "Missile autopilot design using adaptive nonlinear dynamic inversion," in Proc. 2005 American Control Conf., 
Portland, OR, USA, 2005, pp. 3918-3919.

[52] T. Madani and A. Benallegue, "Backstepping sliding mode control applied to a miniature quadrotor flying robot," in Proc. 32nd Annual Conf. on IEEE Industrial Electronics (IECON), Paris, France, 2006, pp. 700-705.

[53] M. Huang, B. Xian, C. Diao, K. Yang, and Y. Feng, "Adaptive tracking control of underactuated quadrotor unmanned aerial vehicles via backstepping," in Proc. 2010 American Control Conf., Baltimore, MD, USA, 2010, pp. 2076-2081.

[54] W. Dong, J. A. Farrell, M. M. Polycarpou, V. Djapic, and M. Sharma, "Command filtered adaptive backstepping," IEEE Trans. Control Systems Technology, vol. 20, no.3, pp.566-580, 2012.

[55] Z. Zuo, "Adaptive trajectory tracking control design with command filtered compensation for a quadrotor," Journal of Vibration and Control, vol. 19, no. 1, pp.94-108, 2012.

[56] T. Yucelen and W. M. Haddad, "Low-frequency learning and fast adaptation in model reference adaptive control," IEEE Trans. Automatic Control, vol. 58, no. 4, pp. 1080-1085, 2013.

[57] N. Hovakimyan and C. Cao, $\mathcal{L}_{1}$ Adaptive Control Theory. Philadelphia, PA: Society for Industrial and Applied Mathematics, 2010 .

[58] I. Kaminer, A. Pascoal, E. Xargay, N. Hovakimyan, C. Cao, and V. Dobrokhodov, "Path following for unmanned aerial vehicles using $\mathcal{L}_{1}$ adaptive augmentation of commercial autopilots" Journal of Guidance, Control, and Dynamics, vol.33, no.2, pp.550-564, 2010.

[59] N. Hovakimyan, C. Cao, E. Kharisov, E. Xargay, and I. M. Gregory, " $\mathcal{L}_{1}$ adaptive control for safety-critical systems guaranteed robustness with fast adaptation" IEEE Control Systems Magazine, vol.31, no. 5, pp. 54-104, 2011.

[60] Z. Zuo and S. Mallikarjunan, " $\mathcal{L}_{1}$ adaptive backstepping for robust trajectory tracking of UAVs" IEEE Trans. Industrial Electronics, vol. 64, no. 4, pp. 2944-2954, 2017.

[61] L. Marconi and R. Naldi, "Robust full degree-of-freedom tracking control of a helicopter," Automatica, vol.43, no.11, pp.1909-1920, 2007.

[62] R. Naldi, L. Gentili, L. Marconi, and A. Sala, "Design and experimental validation of a nonlinear control law for a ducted-fan miniature aerial vehicle," Control Engineering Practice, vol. 18, no. 7, pp. 747-760, 2010.

[63] E. J. J. Smeur, Q. Chu, and G. C. H. E. de Croon, "Adaptive incremental nonlinear dynamic inversion for attitude control of micro air vehicles," Journal of Guidance, Control, and Dynamics, vol.39, no. 3, pp. 450-461, 2016.

[64] E. J. J. Smeur, G. C. H. E. de Croon, and Q. Chu, "Cascaded incremental nonlinear dynamic inversion for MAV disturbance rejection," Control Engineering Practice, vol.73, pp. 79-90, 2018.

[65] L. Marconi, R. Naldi, and A. Isidori, "High-gain output feedback for a miniature UAV," Int. Journal of Robust and Nonlinear Control, vol. 24, no. 6, pp. 1104-1126, 2014.

[66] H. Liu, D. Li, J. Xi, and Y. Zhong, "Robust attitude controller design for miniature quadrotors," Int. Journal of Robust and Nonlinear Control, vol.26, no. 4, pp. 681-696, 2016.

[67] S. Li, J. Yang, W. Chen, and X. Chen, Disturbance Observer-Based Control: Methods and Applications. Taylor \& Francis, 2014.

[68] J. Han, "From PID to active disturbance rejection control," IEEE Trans. Industrial Electronics, vol.56, no. 3, pp.900-906, 2009.

[69] Y. Shtessel, C. Edwards, L. Fridman, and A. Levant, Sliding Mode Control and Observation. Springer New York, 2014.

[70] A. Levant, "Higher-order sliding modes, differentiation and outputfeedback control," Int. Journal of Control, vol.76, no.9-10, pp. 924-941, 2003.

[71] W.-H. Chen, J. Yang, L. Guo, and S. Li, "Disturbance-observerbased control and related methods-an overview," IEEE Trans. Industrial Electronics, vol.63, no. 2, pp. 1083-1095, 2016.

[72] C. Liu, W.-H. Chen, and J. Andrews, "Tracking control of small-scale helicopters using explicit nonlinear MPC augmented with disturbance observers," Control Engineering Practice, vol.20, no.3, pp.258-268, 2012.

[73] H. Lu, C. Liu, L. Guo, and W. H. Chen, "Flight control design for small-scale helicopter using disturbance observer-based backstepping," Journal of Guidance, Control, and Dynamics, vol.38, no.11, pp. 2235-2240, 2015.

[74] L. Besnard, Y. B. Shtessel, and B. Landrum, "Quadrotor vehicle control via sliding mode controller driven by sliding mode disturbance observer," Journal of the Franklin Institute, vol.349, no. 2, pp.658684, 2012.

[75] B. Tian, L. Liu, H. Lu, Z. Zuo, Q. Zong, and Y. Zhang, "Multivariable finite time attitude control for quadrotor UAV: Theory and experimentation," IEEE Trans. Industrial Electronics, vol.65, no.3, pp. 2567-2577, 2018.

[76] L. Wang and J. Su, "Robust disturbance rejection control for attitude tracking of an aircraft," IEEE Trans. Control Systems Technology, vol. 23, no. 6, pp. 2361-2368, 2015.

[77] X. Lyu, J. Zhou, H. Gu, Z. Li, S. Shen, and F. Zhang, "Disturbance observer based hovering control of quadrotor tail-sitter VTOL UAVs using $H_{\infty}$ synthesis," IEEE Robotics and Automation Letters, vol.3, no. 4, pp. 2910-2917, 2018.

[78] K. Ohishi, M. Nakao, K. Ohnishi, and K. Miyachi, "Microprocessorcontrolled DC motor for load-insensitive position servo system," IEEE Trans. Industrial Electronics, vol.IE-34, no. 1, pp. 44-49, 1987.

[79] S. J. Lee, D. Lee, J. Kim, D. Kim, I. Jang, and H. J. Kim, "Fully actuated autonomous flight of thruster-tilting multirotor," IEEE/ASME Trans. Mechatronics, vol.26, no.2, pp.765-776, 2021.

[80] W.-H. Chen, D. Ballance, Gawthrop, and J. O'Reilly, "A nonlinear disturbance observer for robotic manipulators," IEEE Trans. Industrial Electronics, vol. 47, no. 4, pp.932-938, 2000.

[81] X. He, G. Kou, M. Calaf, and K. K. Leang, "In-ground-effect modeling and nonlinear-disturbance observer for multirotor unmanned aerial vehicle control," Journal of Dynamic Systems, Measurement, and Control, vol. 141, no. 7, Article No. 071013, 2019.

[82] K. Guo, J. Jia, X. Yu, L. Guo, and L. Xie, "Multiple observers based anti-disturbance control for a quadrotor UAV against payload and wind disturbances," Control Engineering Practice, vol.102, Article No. 104560, 2020

[83] M. Chen, S. Xiong, and Q. Wu, "Tracking flight control of quadrotor based on disturbance observer," IEEE Trans. Systems, Man, and Cybernetics: Systems, vol.51, no. 3, pp. 1414-1423, 2021.

[84] Y. Yan, J. Yang, C. Liu, M. Coombes, S. Li, and W.-H. Chen, "On the actuator dynamics of dynamic control allocation for a small fixed-wing UAV with direct lift control," IEEE Trans. Control Systems Technology, vol.28, no.3, pp.984-991, 2020.

[85] R. Sanz, P. Garcia, and P. Albertos, "Active disturbance rejection by state feedback: Experimental validation in a 3-DOF quadrotor platform," in Proc. the 54th Annual Conf. of the Society of Instrument and Control Engineers of Japan (SICE), 2015, pp. 794-799.

[86] W. Dong, G.-Y. Gu, X. Zhu, and H. Ding, “A high-performance flight control approach for quadrotors using a modified active disturbance rejection technique," Robotics and Autonomous Systems, vol.83, pp. 177-187, 2016.

[87] H. Yang, L. Cheng, Y. Xia, and Y. Yuan, "Active disturbance rejection attitude control for a dual closed-loop quadrotor under gust wind," IEEE Trans. Control Systems Technology, vol.26, no. 4, pp. 1400-1405, 2018.

[88] C. E. Hall and Y. B. Shtessel, "Sliding mode disturbance observerbased control for a reusable launch vehicle," Journal of Guidance, Control, and Dynamics, vol.29, no. 6, pp. 1315-1328, 2006.

[89] A. Benallegue, A. Mokhtari, and L. Fridman, "High-order slidingmode observer for a quadrotor UAV," Int. Journal of Robust and Nonlinear Control, vol. 18, no.4-5, pp.427-440, 2008.

[90] I. Kaminer, A. Pascoal, E. Hallberg, and C. Silvestre, "Trajectory tracking controllers for autonomous vehicles: an integrated approach to guidance and control," Journal of Guidance, Control, and Dynamics, vol.21, no. 1, pp. 29-38, 1998 
[91] C. Nielsen, C. Fulford, and C. Maggiore, "Path following using transverse feedback linearization: Application to a maglev positioning system," Automatica, vol.46, no.3, pp. 585-590, 2010.

[92] D. R. Nelson, D. B. Barber, T. W. McLain, and R. W. Beard, "Vector field path following for miniature air vehicles," IEEE Trans. Robotics, vol.23, no. 3, pp. 519-529, 2007.

[93] J. Reiner, G. J. Balas, and W. L. Garrard, "Robust dynamic inversion for control of highly maneuverable aircraft," Journal of Guidance, Control, and Dynamics, vol. 18, no. 1, pp. 18-24, 1995.

[94] P. Sun, B. Zhu, Z. Zuo, and M. Basin, "Vision-based finite-time uncooperative target tracking for UAV subject to actuator saturation," Automatica, vol. 130, Article No. Article 109708, 2021.

[95] A. Aguiar and J. Hespanha, "Trajectory-tracking and pathfollowing of underactuated autonomous vehicles with parametric modeling uncertainty," IEEE Trans. Automatic Control, vol.52, no. 8, pp. 13621378, 2007.

[96] E. N. Johnson and S. K. Kannan, "Adaptive trajectory control for autonomous helicopters," Journal of Guidance, Control, and Dynamics, vol. 28, no.3, pp. 524-538, 2005.

[97] A. Aguiar, J. Hespanha, and V. Kokotović, "Path-following for nonminimum phase systems removes performance limitations," IEEE Trans. Automatic Control, vol.50, no. 2, pp. 234-239, 2005.

[98] Z. Zuo, J. Song, and Q.-L. Han, "Coordinated planar path-following control for multiple nonholonomic wheeled mobile robots," IEEE Trans. Cybernetics, 2021. DOI: 10.1109/TCYB.2021.3057335,2021

[99] C. Liu, O. McAree, and W.-H. Chen, "Path-following control for small fixed-wing unmanned aerial vehicles under wind disturbances," Int. Journal of Robust \& Nonlinear Control, vol.23, no. 15, pp. 1682-1698, 2013.

[100] A. Galffy, M. Boeck, and A. Kugi, "Nonlinear 3D path following control of a fixed-wing aircraft based on acceleration control," Control Engineering Practice, vol. 86, pp. 56-69, 2019.

[101] B. Zhu and W. Huo, "3-D path-following control for a model-scaled autonomous helicopter," IEEE Trans. Control Systems Technology, vol. 22, no. 5, pp. 1927-1934, 2014.

[102] Z. Zuo, L. Cheng, X. Wang, and S. K., "Three-dimensional pathfollowing backstepping control for an underactuated stratospheric airship," IEEE Trans. Aerospace and Electronic Systems, vol. 55, no. 3, pp. 1483-1497, 2018.

[103] Z. Zuo, J. Song, Z. Zheng, and Q.-L. Han, "A survey on modelling, control and challenges of stratospheric airships," Control Engineering Practice, vol. 119, Article No. 104979, 2022.

[104] V. Dobrokhodov, I. Kaminer, K. Jones, and R. Ghabcheloo, "Visionbased tracking and motion estimation for moving targets using small UAVs," in Proc. 2006 American Control Conf., 2006, pp. $1428-1433$.

[105] E. Frew, D. Lawrence, and S. Morris, "Coordinated standoff tracking of moving targets using Lyapunov guidance vector fields," Journal of Guidance Control \& Dynamics, vol. 31, no. 2, pp. 290-306, 2008.

[106] H. Chen, K. Chang, and C. S. Agate, "UAV path planning with Tangentplus-Lyapunov vector field guidance and obstacle avoidance," IEEE Trans. Aerospace and Electronic Systems, vol.49, no. 2, pp. 840856, 2013.

[107] A. Pothen and A. Ratnoo, "Curvature-constrained Lyapunov vector field for standoff target tracking," Journal of Guidance Control Dynamics, vol.40, no. 10, pp. 2725-2735, 2017.

[108] Y. Su, Y u, M. J. Gerber, L. Ruan, and T.-C. Tsao, "Nullspace-based control allocation of overactuated UAV platforms," IEEE Robotics and Automation Letters, vol. 6, no. 4, pp. 8094-8101, 2021.

[109] D. Rohr, M. Studiger, T. Stastny, N. R. J. Lawrance, and R. Siegwart, "Nonlinear model predictive velocity control of a VTOL tiltwing UAV," IEEE Robotics and Automation Letters, vol.6, no. 3, pp. 5776-5783, 2021.

[110] W. He, X. Mu, L. Zhang, and Y. Zou, "Modeling and trajectory tracking control for flapping-wing micro aerial vehicles," IEEE/CAA Journal of Automatica Sinica, vol. 8, no. 1, pp. 148-156, 2021.

[111] I. Palunko, Cr uz, and R. Fierro, "Agile load transportation," IEEE
Robotics \& Automation Magazine, vol. 19, no.3, pp. 69-79, 2012.

[112] E. Pounds, D. R. Bersak, and A. M. Dollar, "Stability of small-scale UAV helicopters and quadrotors with added payload mass under PID control," Autonomous Robots, vol.33, no. 1-2, pp. 129-142, 2012.

[113] M. O'Connell, G. Shi, X. Shi, and S.-J. Chung, "Meta-learning-based robust adaptive flight control under uncertain wind conditions," arXiv preprint arXiv: 2103.01932, 2021.

[114] M. Zheng, X. Lyu, X. Liang, and F. Zhang, "A generalized design method for learning-based disturbance observer," IEEE/ASME Trans. Mechatronics, vol.26, no. 1, pp.45-54, 2021.

[115] S. Madruga, A. H. B. M. Tavares, S. O. D. Luiz, T. do Nascimento, and A. M. N. Lima, "Aerodynamic effects compensation on multirotor UAVs based on a neural network control allocation approach," IEEE/CAA Journal of Automatica Sinica, vol.9, no.2, pp.295-312, 2022.

[116] H. Arbabi, "Koopman spectral analysis and study of mixing in incompressible flows," $\mathrm{PhD}$ Thesis, University of California-Santa Barbara, 2017

[117] M. Korda and I. Mezić, "Linear predictors for nonlinear dynamical systems: Koopman operator meets model predictive control," Automatica, vol.93, pp. 149-160, 2018.

[118] Z. Zuo and C. Wang, "Adaptive trajectory tracking control of output constrained multi-rotors systems," IET Control Theory \& Applications, vol. 8, no. 13, pp. 1163-1174, 2014.

[119] Y. Liu, Z. Meng, Y. Zou, and M. Cao, "Visual object tracking and servoing control of a nano-scale quadrotor: System, algorithms, and experiments," IEEE/CAA Journal of Automatica Sinica, vol. 8, no. 2, pp. 344-360, 2021.

[120] W. Youn, M. B. Rhudy, A. Cho, and H. Myung, "Fuzzy adaptive attitude estimation for a fixed-wing UAV with a virtual SSA sensor during a GPS outage," IEE Sensors Journal, vol.20, no.3, pp. 1456-1472, 2020.

[121] H. Choi and Y. Kim, "Reactive collision avoidance of unmanned aerial vehicles using a single vision sensor," Journal of Guidance, Control, and Dynamics, vol. 36, no. 4, pp. 1234-1240, 2013.

[122] B. Wang, J. Xie, and J. Chen, "Data-driven multi-uav navigation in large-scale dynamic environments under wind disturbances," in AIAA SciTech Forum, Virtual Event, AIAA 2021-1284, DOI: 10.2514/6.2021-1284, 2021, pp. 1-13.

[123] S. Givigi and T. Jardine, "Machine learning for data-driven control of robots," IEEE Potentials, vol.37, no. 4, pp. 35-39, 2018.

[124] V. Mnih, K. Kavukcuoglu, D. Silver, A. A. Rusu, J. Veness, M. G. Bellemare, A. Graves, M. Riedmiller, A. K. Fidjeland, G. Ostrovski, S. Petersen, C. Beattie, A. Sadik, I. Antonoglou, H. King, D. Kumaran, D. Wierstra, S. Legg, and D. Hassabis, "Human-level control through deep reinforcement learning," Nature, vol.518, pp. 529-533, 2015.

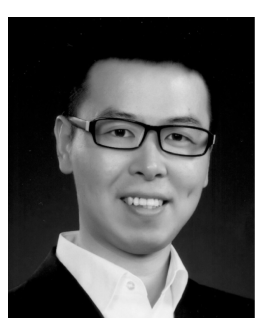

Zongyu Zuo (Senior Member, IEEE) received his B.Eng. degree in automatic control from Central South University, Hunan, China, in 2005, and Ph.D. degree in Control Theory and Applications from Beihang University (BUAA), Beijing, China, in 2011.

He was an academic visitor at the School of Electrical and Electronic Engineering, University of Manchester from September 2014 to September 2015 and held an inviting associate professorship at Mechanical Engineering and Computer Science, UMR CNRS 8201, Université de Valenciennes et du Hainaut-Cambrésis in October 2015 and May 2017. He is currently a full professor at the School of Automation Science and Electrical Engineering, Beihang University. His research interests are in the fields of nonlinear system control, control of UAVs, and coordination of multi-agent system. He was identified as a Highly Cited Researcher - 2020 by Clarivate Analytics.

Prof. Zuo serves as an Associate Editor of the Journal of The Franklin Institute, the Journal of Vibration and Control, the International Journal of Aeronautical \& Space Sciences, and the International Journal of Digital Signals and Smart Systems. He also serves as a member of the early career advisory board of the IEEE/CAA Journal of Automatica Sinica. 


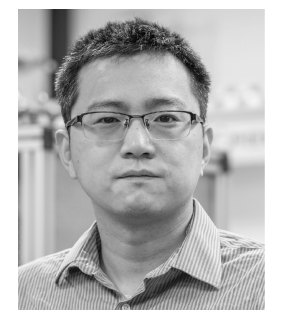

Cunjia Liu received Ph.D. degree in autonomous vehicle control from Loughborough University in 2011. He was a research associate with the Department of Aeronautical and Automotive Engineering at Loughborough University, where he became a lecturer in 2013 and currently holds a Reader position in Unmanned Vehicles. He has a strong background in flight control, autonomous vehicles, and Bayesian estimation. His recent work focuses on the novel perception and decision algorithms for autonomous vehicles and their applications in security, industry and agriculture domains.

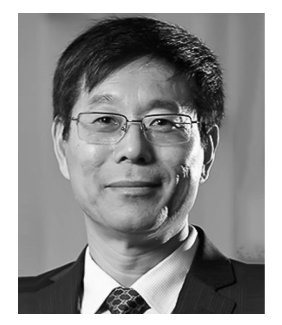

Qing-Long Han (Fellow, IEEE) received the B.Sc. degree in Mathematics from Shandong Normal University, Jinan, China, in 1983, and the M.Sc. and $\mathrm{Ph} . \mathrm{D}$. degrees in Control Engineering from East China University of Science and Technology, Shanghai, China, in 1992 and 1997, respectively.

Professor Han is Pro Vice-Chancellor (Research Quality) and a Distinguished Professor at Swinburne University of Technology, Melbourne, Australia. He held various academic and management positions at Griffith University and Central Queensland University, Australia. His research interests include networked control systems, multi-agent systems, time-delay systems, smart grids, unmanned surface vehicles, and neural networks.

Professor Han was the recipient of The 2021 Norbert Wiener Award (the Highest Award in systems science and engineering, and cybernetics), The 2021 M. A. Sargent Medal (the Highest Award of the Electrical College
Board of Engineers Australia), The 2021 IEEE/CAA Journal of Automatica Sinica Norbert Wiener Review Award, The 2020 IEEE Systems, Man, and Cybernetics (SMC) Society Andrew P. Sage Best Transactions Paper Award, The 2020 IEEE Transactions on Industrial Informatics Outstanding Paper Award, and The 2019 IEEE SMC Society Andrew P. Sage Best Transactions Paper Award.

Professor Han is a Member of the Academia Europaea (The Academy of Europe) and a Fellow of The Institution of Engineers Australia. He is a Highly Cited Researcher in both Engineering and Computer Science (Clarivate Analytics, 2019-2021). He has served as an AdCom Member of IEEE Industrial Electronics Society (IES), a Member of IEEE IES Fellow Committee, and Chair of IEEE IES Technical Committee on Networked Control Systems. He is Co-Editor-in-Chief of IEEE TRANSACTIONS ON INDUSTRIAL INFORMATICS, Deputy Editor-in-Chief of IEEE/CAA JOURNAL OF AUTOMATICA SINICA, Co-Editor of Australian Journal of Electrical and Electronic Engineering, an Associate Editor for 12 international journals, including the IEEE TRANSACTIONS ON CYBERNETICS, IEEE INDUSTRIAL ELECTRONICS MAGAZINE, Control Engineering Practice, and Information Sciences, and a Guest Editor for 13 Special Issues.

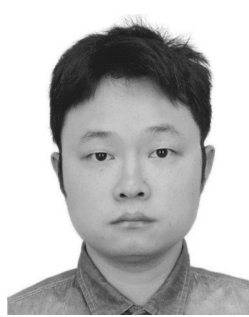

Jiawei Song received the B.Eng. degree in automation from Ocean University of China, Shandong, China, in 2013, and the MA.Eng degree in control theory and applications from Beihang University (BUAA), Beijing, China, in 2018. He is currently working toward the Ph.D degree in control theory and applications with Beihang University. His research interests are in the fields of nonlinear system control and control of UAVs. 